\title{
JACOPO BARSOTTI
}

Nel segno di un profondo rimpianto ricordo lamico Jacopo Barsotti, che ci ha lasseiato il 27 Ottobre 1987; era nato a Torino il 28 Aprile 1921.

Se risalgo nel tempo lo rivedo a Roma, assistente di geometria, da poco laureato alla Normale di Pisa. Già lavorava in piena autonomia, con originalità di interessi, valendosi di un supporto culturale non comune.

In quegli anni i metodi dell'algebra moderna in geometria algebrica erano in Italia pressochè sconosciuti: a Roma c'era solo Barsotti che già li padroneggiava con sicura maestria.

Fu fra i pochi allora ad avvertire la necessità di incontrarsi con le scuole matematiche più avanzate, da cui l'Italia era rimasta separata per molti anni, causa i ben noti motivi bellici e politici. Per questo, ottenuta una "fellowship" dall'Università di Princeton, partì per gli Stati Uniti, dove rimase molti anni, divenendo "full professor" all'Università di Pittsburgh.

Tornerà in Italia nel '60, vincitore di un concorso. Chiamato a Pisa, dove rimarrà fino al ' 68 , fu uno degli animatori di quel periodo magico della scuola matematica pisana, che, in molti, non solo della mia generazione, ha lasciato tracce profonde.

Dal '68 exa passato all'Università di Padova e anche qui la sua influenza fu subito evidente.

Barsotti non è stato allievo di alcun maestro, anche se nei suoi ricordi era presente Cherubino, che probabilmente ispirò i suoi primi lavori. Ben presto però, Barsotti passau a interessarsi di varietà abeliane e più in generale di varietà gruppali in caratteristica zero e positiva: argomento che, da sempre, è di importanza centrale nella matematica, attraendo, da Abel e Jacobi in poi, i matematici più illustri. Barsotti lascia in queste teorie una traccia importante. I suoi teoremi sulla struttura delle varietà gruppali sono definitivi.

limitandoci qui ai contributi di assoluto rilievo scientifico, vanno ricordati i settie capitoli di "Metodi analitici per varietà abeliane in caratteristica positiva" pubblicati dal '64 al '66 sugli "Annali della Scuola Normale" di Pisa. In questo imponente lavoro, Barsotti costruisce dei gruppi analitici associati a varietà abeliane (i gruppi di Barsotti-Tate) a partire dai quali ottiene quella che poi si chiamerà la coomologia cristallina. 